\title{
Consideration Factors in Application of Thermocouple Sensors for RCS Temperature Instrumentation
}

\author{
Inkoo Hwang*, Sewoo Cheon, Wonman Park \\ I\&C and HFE Research Division, Korea Atomic Energy Research Institute, 989-111, \\ Daedeok-daero, Yuseong-gu, Daejeon 305-353, Republic of Korea \\ * ikhwang@kaeri.re.kr
}

\begin{abstract}
Because of harsh radiated environmental conditions, it is necessary to use thermocouples (TCs) in the temperature instrumentation channels of a reactor coolant system (RCS) in an integrated pressurized water reactor vessel. Conventionally, resistance temperature detectors (RTDs) have been adopted for RCS temperature measurement.

Therefore, we have conducted an analysis and review of instrumentation error factors in the measurement circuits of RTD and $\mathrm{TC}$ sensors to specify the influence on measurement accuracy for application of TCs instead of RTDs for RCS temperature instrumentation.

From the review and analysis results, it is anticipated that a measurement accuracy deterioration would be an issue and that a drift range should be investigated for the anticipated operational temperature conditions.
\end{abstract}

Keywords—RTD Measurement Circuits, TC Accuracy

\section{INTRODUCTION}

Resistance temperature detectors (RTDs) and K-type thermocouples (TCs) are the most common devices for measuring the temperature of fluid in nuclear power plants (NPPs). RTDs are typically used for measuring reactor coolant system (RCS) temperature because an accurate and stable sensing is needed for calculating the thermal output power and regulating the reactor during a normal operation. In some instances, however, TCs are favored over RTDs for measuring RCS temperature, as in the case of a core inlet or outlet of an integrated reactor. RTD-based temperature circuits are generally regarded as very accurate and stable in comparison with TC-based circuits, however, there are some considerable factors diminishing the measurement accuracy depending on the measurement methods and calibration circumstances in temperature instrumentation systems.

Thus, this paper introduces the typical factors affecting the measurement errors and specifies the anticipated inaccuracy sizes due to each factor in RTD-based and TC-based temperature instrumentation channels in Section II and III. Based on the investigated inaccuracy numbers, the total sizes of uncertainty deterioration in the application of TCs instead of RTDs are calculated in Section IV. Section V includes the summary of this paper.

\section{FACTORS AFFECTING THE ERRORS IN RTD-BASED CIRCUITS AND RELIEVING COUNTER-MEASURES}

Although RTDs are commonly used for temperature measurement bellow $500{ }^{\circ} \mathrm{C}$ in industrial process plants such as NPPs, there are many factors to be considered regarding the measurement accuracy in RTD instrumentation channels. Such factors include lead wire resistance effect, self-heating effect, electromagnetic force error, non-linearity, corrosion, radiation, and insulation loss [1-3]. This section describes the sizes of each effect in the key factors among the above examples, which we have investigated in the previous research [3].

\section{A. Lead Wire Resistance Effects}

The effect of resistance of the lead wires is not negligible, even in the case where the distance between RTD sensors and instrumentation circuits is a couple of meters. This is because of the low sensitivity characteristic of $100 \Omega$-RTDs, called Pt- 100 . The sensitivity (temperature coefficient) is only $0.385 \Omega /{ }^{\circ} \mathrm{C}$. Accordingly, a $10 \mathrm{~m}$ wire of $20 \mathrm{AWG}(=0.33 \Omega)$ contributes an error of $0.84^{\circ} \mathrm{C}$.

Therefore, in industrial RTD instrumentation systems, it is necessary to use a compensation method to remove this wire resistance effect for measuring the process temperature correctly.

A simple way to compensate for the wire resistance effect is to adjust the process temperature reading value in the RTD instrumentation system by a proper in-situ calibration.

However, if the resistance of the lead wires were to change because of the ambient temperature difference from the in-situ calibrated condition, then a temperature reading error dependent on the degree of temperature variation would occur. Should the temperature of the lead wire be increased to $35{ }^{\circ} \mathrm{C}$ from $10{ }^{\circ} \mathrm{C}$, the resistance of a $300 \mathrm{~m}$ wire of $20 \mathrm{AWG}$ size 
would increase by $10 \%$; that is, from $10 \Omega$ to $11 \Omega$. This change would create a reading error of $5.2{ }^{\circ} \mathrm{C}$ in a 2 -wired pt-100 RTD circuit.

Thus, in the case of industrial RTD-based temperature instrumentation systems, it is necessary to use a technical solution for reducing or eliminating the effect of wire resistance change. However, such a solution often requires the use of at least one extra lead wire from the sensor to the instrumentation electronics.

The typical solutions avoiding the wire resistance effect are (a) 3-wired bridge connection method, (b) 3-wired 1-curent injection method, (c) 3-wired 2-current injection method, $(d)$ 4-wired 1-current injection method, and (e5) local transmitter method.

Usually, a properly designed 3-wired connection circuit can cancel the wire resistance effect completely. In particular, 3-wired with 2-current or 4-wired methods are widely adopted to consistently eliminate the lead wire resistance effect in the industrial RTD-based temperature instrumentation systems.

Accordingly, an appropriate choice of cancellation method for wire resistance can ensure that any error due to a change in lead wire resistance will be negligible.

\section{B. Self-heating Effects}

In all RTD-based temperature measurement systems, an excitation current flows though the RTD sensor element. Thus, it is possible to measure the resistance of the RTD and convert the resistance to the medium temperature value. The excitation current flowing through the RTD element generates a Joule's heat energy, which results in a temperature increment beyond the medium temperature to be measured. This additional increment in temperature creates an error with regard to the measurement value.

The error due to this self-heating effect can be expressed as follows:

$$
\Delta T=\left(I^{2} \times R_{r t d}\right) \times h
$$

where, $\Delta T$ is the change in temperature due to the power dissipation, $I$ is the excitation current of the RTD sensor element, $R_{r t d}$ is the resistance of the RTD sensor element in $\Omega$, and $h$ is the self-heating coefficient in ${ }^{\circ} \mathrm{C} / \mathrm{mW}$.

To minimize the error due to self-heating, the excitation current, RTD resistance, and $h$ should be reduced. In this regard, a pt-100 is the most suitable choice because of its low resistance. The size of $h$ is dependent on the surface that is in contact with the medium, the heat transfer coefficient, and the temperature gradient from the RTD sensor element.

Therefore, the size of the excitation current is only the practically manageable parameter to decrease the self-heating effect.

For an example, if we take $0.05{ }^{\circ} \mathrm{C} / \mathrm{mW}$ to be a typical self-heating coefficient of an RTD sensor, considering that its range is from $0.01{ }^{\circ} \mathrm{C} / \mathrm{mW}$ to $0.1{ }^{\circ} \mathrm{C} / \mathrm{mW}$ in water, then the temperature deviation, $\Delta T$, will be $+1.05{ }^{\circ} \mathrm{C}$ when the excitation current is $10 \mathrm{~mA}$ and the process temperature is $300{ }^{\circ} \mathrm{C}$. However, $\Delta T$ will decrease to $+0.0105{ }^{\circ} \mathrm{C}$ when $1 \mathrm{~mA}$ of current is applied. IEC 60751[4] recommends an excitation current of less than $1 \mathrm{~mA}$.

\section{Thermal EMF errors}

If there is inhomogeneity in material composition along the RTD connection metal wires, the dissimilar metal constituents will act as noisy TCs in the RTD circuit. These unintended TCs will then generate error voltages related to temperature gradients. This inhomogeneity in RTD connection wire metals can be caused from the manufacturing process or material change due to oxidation, etc. during the long operation period of an RTD system, particularly in high temperature applications.

The materials of the RTD element wires, internal lead wires, and external connection lead wires are usually different from each other. In general, the RTD element wires are made of pure platinum; the internal wires, a platinum or a gold alloy: and the external connection lead wires, copper [5].

Fig. 1 illustrates the potential unintended TC parts in an RTD circuit. If the temperature at one end of an electric conductor differs to that at the other end, then a thermal electromagnetic force $(\mathrm{EMF})$ arises in proportion to the temperature difference because of the Seebeck effect.

In Fig. 1, the total EMF along an RTD instrumentation circuit can be defined as follows:

$$
E M F=E 1+E 2+E 3+E 4+E 5
$$

In Eq. (2), the total thermoelectric EMF is not zero when there is a temperature deviation between $T_{1}$ and $T_{2}$, or between $\mathrm{T}_{3}$ and $\mathrm{T}_{4}$, or $\mathrm{T}_{5}$ and $\mathrm{T}_{6}$, or if there is a deviation in homogeneity between the materials of the paired two lead wires. This unnecessary EMF voltage yields a small error in the RTD resistance reading of the voltmeter in this circuit.

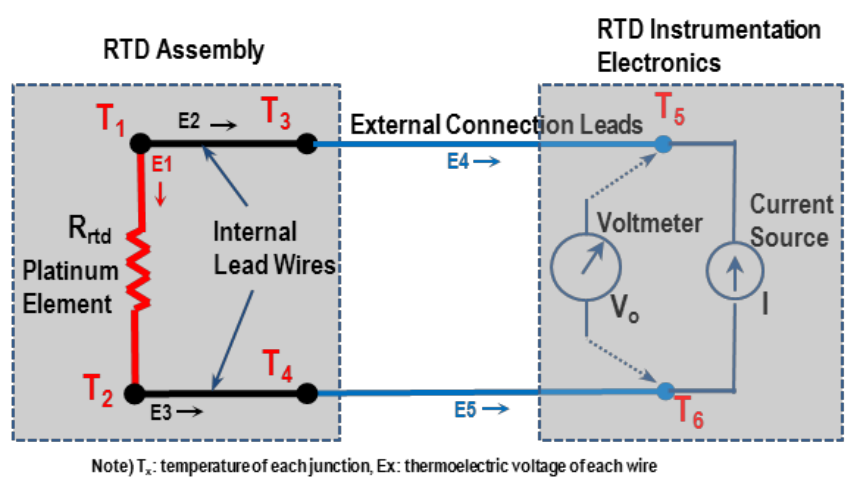

Fig. 1 Unintended TC elements in RTD connection wires

A research has shown that the EMF voltages lie within the range $\pm 0.1 \sim 17.9 \mu \mathrm{V}$ for 25 nuclear grade RTDs at $300{ }^{\circ} \mathrm{C}$ [7]. 
The maximum value, $\pm 17.9 \mu \mathrm{V}$, corresponds to an error of $\pm 0.047{ }^{\circ} \mathrm{C}$ when $\mathrm{I}=1 \mathrm{~mA}$ in the case of Pt-100 RTD. If the excitation current source, i.e. I in Fig. 1, is small, then the error will be smaller.

\section{Accuracy of Instrumentation Electronics}

To obtain the process temperature signal from the RTD resistance, there must be an electronic or other type of readout instrument capable of converting a resistance signal to a corresponding temperature value. In most industrial process plants, the instrumentation electronics are implemented as a part of a digital control system.

The accuracy ranges of industrial RTD signal conversion modules are within $\pm 0.1 \sim 0.5 \%$ of full scale. One such device currently in use for NPPs has an accuracy of $\pm 0.3 \%$. If we narrow the full span of measurement range to be $250 \sim 350{ }^{\circ} \mathrm{C}$ in the signal conversion module to obtain a better accuracy for RCS temperature application, the accuracy limit will be approximately $\pm 0.5{ }^{\circ} \mathrm{C}$, considering other factors related to accuracy such as power supply effect, stability(drift) effect, etc.

\section{FACTORS AfFeCting THE ERRORS IN TC-BASED TEMPERATURE MEASUREMENT CIRCUITS}

Although TCs are simple and low-cost sensing elements for measuring fluid or gas temperature at high temperature ranges, having a fast response time performance, instrumentation designers are reluctant to use TCs in control and precise monitoring systems in NPPs, owing to accuracy and stability issues compared to RTDs.

However, because RTDs are not suitable for use inside a nuclear reactor vessel, TCs are the only choice for measuring the temperature of reactor coolant when the temperature sensors need to be installed inside the reactor vessel in an integrated reactor system. Accordingly, we analyzed the extent of accuracy deterioration when TCs are used instead of RTDs for RCS temperature measurement [6].

This section summarizes the key error sources and their associated sizes of that analyzed in Reference [6].

\section{A. Overview of Error Sources in a TC instrument Channel}

Fig. 2 illustrates a typical TC instrument channel block diagram, which is generally used for instrumentation and control (I\&C) systems in industrial plants such as NPPs. We have classified each error factor present in the measurement loop depicted in the figure with the circled numbers, i.e. (1) process medium error, (2) error in the TC itself, (3) connector error, (4) extension wire error, (5) instrumentation electronic device error, and (6) cold junction sensing error. Note that there are other error factors that affect the total uncertainty of measurement; for example, drift error and external noise ingress. However, these are not depicted in Fig. 2 for simplicity in analysis.

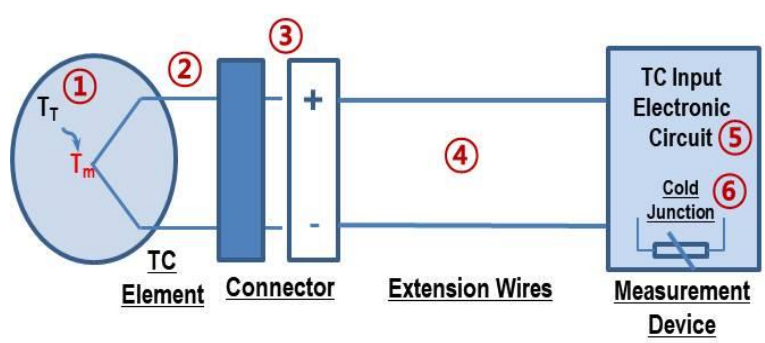

Fig. 2 Error sources in a Thermocouple connection circuit

\section{B. Analysis of Error Terms}

The first error factor, (1), indicates the process medium error, which means a deviation between the true process temperature, $\mathrm{T}_{\mathrm{T}}$, and the temperature transferred to the measurement junction of $\mathrm{TC}, \mathrm{T}_{\mathrm{m}}$. This type of error is related to the installation and junction types of TCs. It may be large or small depending on the installed situations and locations. However, for our comparison analysis, it is assumed negligible.

The second error source, (2), is an inherent manufactured error in a TC itself and a dominant factor in the aspect of accuracy in a TC-based temperature instrument. For a K-type $\mathrm{TC}$, which is widely used in industrial plants, the error limit of Class 2 is specified as $\pm 2.5{ }^{\circ} \mathrm{C}[7]$. If we take Class 1 for better accuracy performance, the error limit becomes $\pm 1.5{ }^{\circ} \mathrm{C}$

The third piece, (3), is an error resulting from temperature difference, $\Delta \mathrm{T}$, along the $\mathrm{TC}$ connector pins. It is anticipated that the close proximity of the connector pins would not make $\Delta \mathrm{T}$ more than $\pm 0.2{ }^{\circ} \mathrm{C}$.

The fourth error factor, (4), is an inherent manufactured error in the extension wires, which must be compatible to the TC element type. For a K-type extension wire, the error limit of Class 2 is specified as $\pm 2.5^{\circ} \mathrm{C}$ and the error limit of Class 1 is specified $\pm 1.5^{\circ} \mathrm{C}[8]$.

The fifth error source, (5), represents the total uncertainty in the TC signal conversion process, which converts the millivolt signal of the TC to a corresponding temperature value. Assuming that the signal conversion accuracy is the same as the RTD signal conversion module described in Section II-D, the error limit will be $\pm 0.5{ }^{\circ} \mathrm{C}$.

The sixth part, (6), indicates the error of the reference junction temperature, which must be precisely measured for calculating the medium temperature. The reference junction temperature is measured by a high-resolution thermometer such as a thermistor. Therefore, it is reasonable to regard its uncertainty range as $\pm 0.5^{\circ} \mathrm{C}$.

Although drift or instability error caused by atomic crystal structure change during a long period of usage is of concern with regard to TC application, we have disregarded the error in our accuracy comparison analysis because the range of drift is highly variable depending on the temperatures in which TCs 
are exposed, TC structures (bare-wire or mineral-insulated sheath types), and manufacturers [3].

\section{UNCERTAINTY CALCULATIONS}

With the error sizes included in Sections III and IV, we have calculated the overall uncertainty of the TC-based and RTD-based measurement channels for comparison via the root-sum-square (RSS) method [9, 10]. Excluding the process medium error and drift error, the overall uncertainty of a TC-based system, for Class 2 TCs and extension wires, is as follows:

$$
\begin{aligned}
U_{T C-\text { class } 2}= & \pm\left(2.5^{2}+0.2^{2}+2.5^{2}+0.5^{2}+0.5^{2}\right)^{\frac{1}{2}} \\
& \approx \pm 3.6^{\circ} \mathrm{C}
\end{aligned}
$$

Correspondingly, for Class 1 TCs and extension wires, it is:

$$
\begin{aligned}
U_{T C-\text { class } 1}= & \pm\left(1.5^{2}+0.2^{2}+1.5^{2}+0.5^{2}+0.5^{2}\right)^{\frac{1}{2}} \\
& \approx \pm 2.3^{\circ} \mathrm{C}
\end{aligned}
$$

In the case of RTD systems, if we assume the use of a 3-wired or 4-wired perfect wire resistance compensation circuit, the error factors are the process medium error, RTD element error, self-heating error, thermal EMF effect, drift error, and the conversion error of the signal conversion module. The tolerance of RTD element is specified in a national standard [11]. The tolerance value is $\pm 1.8^{\circ} \mathrm{C}$ at $300^{\circ} \mathrm{C}$ for Class $\mathrm{B}$ and $\pm 0.75^{\circ} \mathrm{C}$ at $300^{\circ} \mathrm{C}$ for Class $\mathrm{A}$.

Thus, excluding the process medium error and drift error, the overall uncertainty of an RTD-based system, for Class B, is as follows:

$$
\begin{aligned}
U_{R T D-\text { class } B} & = \pm\left(1.8^{2}+0.047^{2}+0.5^{2}\right)^{\frac{1}{2}}+0.01 \\
& \approx \pm 1.88^{\circ} \mathrm{C}
\end{aligned}
$$

Correspondingly, for Class A, it is:

$$
\begin{aligned}
U_{R T D-\text { class }} & = \pm\left(0.75^{2}+0.047^{2}+0.5^{2}\right)^{\frac{1}{2}}+0.01 \\
& \approx \pm 0.91^{\circ} \mathrm{C}
\end{aligned}
$$

In Eqs. (5) and (6), the self-heating error is taken as +0.01 because it is always a positive side error.

With comparison of the uncertainty values from Eq. (3) to Eq. (6), it is concluded that the uncertainty range will increase considerably when TCs are used for measuring RCS temperature instead of RTDs. If we consider the drift factor of TCs, the difference will increase further.
Therefore, the usage of TCs instead of RTDs will bring up some loss of margin in set-point determination for the reactor protection system.

\section{CONCLUSION}

This paper addressed the issue of accuracy with regard to the use of K-type TCs for RCS temperature measurement in an integrated reactor. With a comprehensive review and analysis of error factors of both RTD-based and TC-based temperature instrumentation circuits, we have estimated the extent of accuracy loss when TCs are used instead of RTDs.

It has been identified that the measurement uncertainty will increase considerably when TCs are adopted for RCS temperature measurement.

A concern of this paper is that it is not easy to confine drift error tolerance of TC elements, because the drift mechanism is complicated and variable depending on the temperatures in which TCs are exposed and the mechanical and material properties of TCs. In contrast, RTDs generally have a small drift value.

Based on our review and analysis, it can be concluded that the highest accuracy class of TCs and extension wires should be utilized for improving measurement accuracy. Moreover, an experiment should be conducted to investigate the drift error range of the TCs to be used for the normal operating temperature condition during their required operation period.

\section{REFERENCES}

[1] Acromag Inc., Criteria for Temperature Sensor Selection of T/C and RTD Sensor Types: The Basics of Temperature Measurement Using RTDs, Part 1 3 of 3, May 2011.

[2] Rudi Van Nieuwenhove and Ludo Vermeeren, Irradiation effects on temperature sensors, R-3693, ref. INSTR/1248/03-02, March 2003.

[3] Inkoo Hwang, et al., "Considerations regarding Temperature Measurement using RTDs for Industrial Process Plants," KNS Autumn Meeting, Yeosu, Korea, Oct. 25-26, 2018.

[4] IEC 60751, Industrial Platinum Resistance Thermometers and Platinum Temperature Sensors, Ed., 2.0, 2008

[5] Hayashi Denko Inc., Characteristics of RTD Elements, http://hayashidenko.co.jp/en/RTDcharacteristics.html

[6] Inkoo Hwang, et al., "Review of Error Sources for Application of Thermocouples in RCS Temperature Measurement for an Integrated Reactor," to be presented in KNS Spring Meeting, Jeju, Korea, May. 23-24, 2019.

[7] KS C IEC 60584-2, Thermocouples - Part 2: Tolerances, 2004.

[8] KS C IEC 60584-3, Thermocouples - Part 3: Extension and compensation cables - Tolerances and identification system, 2004.

[9] ASME PTC 19.1-1998, Test Uncertainty, Instruments and Apparatus, Supplement to the ASME Performance Test Codes, 1998.

[10] James T. Nakos, Uncertainty Analysis of Thermocouple Measurements Used in Normal and Abnormal Thermal Environment Experiments at Sandia's Radiant heat Facility and Lurance Canyon Burn Site, Sandia Report, Apr. 2004.

[11] KS C 1616, Resistance Temperature Detector, 1992(2017 Reaffirmed), Dec. 1992. 